\title{
Screening and prevention of neonatal glucose 6-phosphate dehydrogenase deficiency in Guangzhou, China
}

\author{
J. Jiang', B. Li' ${ }^{2}$ W. Cao ${ }^{2}$, X. Jiang ${ }^{2}$, X. Jia ${ }^{2}$, Q. Chen ${ }^{2}$ and J. Wu ${ }^{2}$ \\ ${ }^{1}$ Guangdong Women and Children Hospital, Guangzhou, China \\ ${ }^{2}$ Neonatal Screening Center of Women and Children Medical Center, \\ Guangzhou, China
}

Corresponding author: J. Jiang

E-mail: jianhuijiang@yeah.net

Genet. Mol. Res. 13 (2): $4272-4279$ (2014)

Received May 21, 2013

Accepted October 4, 2013

Published June 9, 2014

DOI http://dx.doi.org/10.4238/2014.June.9.13

\begin{abstract}
We aimed to summarize the results of screening protocol and prevention of neonatal glucose 6-phosphate dehydrogenase (G6PD) deficiency during a 22-year-long period to provide a basis of reference for the screening of this disease. About 1,705,569 newborn subjects in Guangzhou City were screened for this deficiency. Specimens were collected according to the conventional method of specimen acquisition for "newborn dried bloodspot screening", preserved, and inspected. The specimens were studied with fluorescent spot test and quantitative fluorescence assay. Diagnosis was performed using the modified NBTG6PD/6PGD ratio method. Bloodspot filter paper specimens were sent to the laboratory within $24 \mathrm{~h}$ via EMS Express, and the G6PD test was performed on the same day. The G6PD deficiency-positive rate was $4.2 \%$ in the samples screened using the fluorescent spot test, while it was $5 \%$ in case of the quantitative fluorescence assay. Neonatal screening for G6PD deficiency for 11,437 cases (6117 boys and 5320 girls) showed positive results in 481 cases. About 420 cases ( 318 boys and 102 girls) of G6PD deficiency were confirmed with the modified
\end{abstract}


Duchenne NBT ratio method. The total detection rate was 3.7:5.2\% for boys and $1.9 \%$ for girls. Quantitative fluorescence assay improved the sensitivity and detection rate. Accelerating the speed of sample delivery by using Internet network systems and ensuring online availability of screening results can aid the screening and diagnosis of this deficiency within 1 week of birth.

Key words: Glucose 6-phosphate dehydrogenase; Deficiency; Neonatal screening

\section{INTRODUCTION}

Glucose 6-phosphate dehydrogenase (G6PD) deficiency is a global disease affecting multi-ethnic populations with red blood cell enzyme defects and hemolytic anemia; more than 4 million patients worldwide suffer from this deficiency. Southeast Asia, the Middle East, and China are prone to this disease. The south and southwest regions of China show the highest incidence rate of $5-10 \%$.

In 2010, the question “G6PD Screening: Is it really required?" (Foroughinia and Karimi, 2007; Kaur et al., 2010; Kuwahata et al., 2010; Parashar, 2010) was put forth. One main reason contributing to the severity of this disease is the fact that acute hemolysis can occur and that it is difficult to prevent the hemolysis caused by accidental intake of drugs. Most patients with G6PD deficiency had no experience of acute hemolysis. We intended to identify whether the neonatal screening for G6PD deficiency is important, the role of neonatal screening in diagnosis, and the critical points to be considered in this process.

We studied a newborn screening program for G6PD deficiency, which was initiated in 1989 in Guangzhou, China, and 1,705,569 newborns were screened until September 30, 2011.

\section{MATERIAL AND METHODS}

\section{Subjects}

Around 1,705,569 neonatal subjects were enrolled for the screening from 1989 to 2011 (September) from Guangzhou, China. This study was conducted according to the guidelines of the Declaration of Helsinki. This study was approved by the Ethics Committee of Neonatal Screening Center of Women and Children Medical Center in Guangzhou. Written informed consent was obtained from the parents of all participants.

\section{Specimens}

Newborn dried bloodspot screening specimens were used. Screening specimens were collected and preserved according to the conventional method of specimen acquisition for "newborn dried bloodspot screening", and delivered to the newborn screening center of Guangzhou City within 3-5 days. About 1,210,308 blood film specimens were delivered by registered post before March 2009 and 495,261 blood film specimens were delivered after April 2009 by EMS. 


\section{Laboratory assays}

In the fluorescent spot test used from 1989 to 2001 (September), spots of fluorescence on the filter paper were observed with the naked eye. Disappearance or weakening of fluorescence was considered a positive test for G6PD deficiency. About 461,801 samples were screened. Quantitative fluorescence assay was used from October 2002 to March 2003 (Johnson et al., 2009), A G6PD level of $<2.0 \mathrm{U} / \mathrm{gHb}$ was considered positive for G6PD deficiency. About 41,714 samples were screened with this assay. About 1,202,054 samples were screened using a combination of qualitative and quantitative methods of detection from April 2003 to September 2011.

\section{Quality control of experiments}

The inter-laboratory quality control policy followed in the National Yang-Ming University was used by us. About 420 pieces of the dried bloodspot specimens were used for quality control in this study, including 170 pieces as positive control and 250 pieces as negative control.

\section{G6PD deficiency confirmatory test}

The modified Du's NBT ratio method was used. The results of the screening program were sent via mail, telephone, and Internet network systems. Accelerating the speed of sample delivery by using Internet network systems and ensuring online availability of screening results can aid the screening and diagnosis of this deficiency.

\section{Reporting methods}

In order to accelerate the speed of sample delivery, the results were published online by using the internet network system of "Guangzhou newborn screening information system for inherited metabolic disease", which was developed by independent research and development in April 2009.

\section{RESULTS}

\section{Fluorescent spot test}

The G6PD deficiency-positive rate was found to be $4.2 \%$.

\section{Quantitative fluorescence assay}

About 1466 pieces of dried bloodspot specimens were detected within 7 days. The G6PD activity was $4.08 \pm 1.27 \mathrm{U} / \mathrm{g} \mathrm{Hb}$ and the fifth percentile value was $2.00 \mathrm{U} / \mathrm{gHb}$. Screening by this assay showed that the G6PD deficiency-positive rate was 5\% when the value of G6PD was below $2 \mathrm{U} / \mathrm{g} \mathrm{Hb}$ as the positive tangent value. The results of continuous detection of 2172 pieces of dried bloodspot specimens indicated that the G6PD activity decreased by 
$32 \%$ at 7 days and by more than $50 \%$ at 14 days, suggesting that this activity declined naturally with increase in the detection time.

\section{G6PD deficiency confirmatory test}

A group of 11,437 cases screened for the deficiency ( 6117 boys and 5320 girls) was further checked using the modified Du's NBT ratio method. About 420 cases ( 318 boys and 102 girls) of G6PD deficiency were confirmed. The total detection rate was $3.70 \%$ (5.20\% for boys and $1.90 \%$ for girls).

\section{Molecular biology of G6PD}

DNA was directly amplified from the dried bloodspot specimens of 168 cases (boys) of G6PD deficiency, followed by restriction endonuclease analysis. We found 5 of the 11 kinds of point mutation of G6PD gene to be present in the Chinese population.

\section{Quality control of G6PD screening tests}

About 420 pieces of the dried bloodspot specimens were used for quality control in this study and we found that 172 pieces gave positive results and 2 pieces gave false-positive results. The rate of false-positive results was $1.2 \%$. No false-negative results were noted.

\section{Publishing of G6PD screening results online}

Since November 2000, the results of G6PD screening, including positive and negative results, were uploaded on the "neonatal screening software" by the Neonatal Screening Center and transmitted to the server for storage, followed by real-time transmission through Internet network system of the Guangzhou Women and Children's Medical Center. "Guangzhou newborn screening information system for inherited metabolic disease" was developed by independent research and development in April 2009.

The screening results can be immediately uploaded on the specified network server. Maternity hospitals can access the website (www.gznsn.net) to query the G6PD screening results of their own hospitals and to print the report. Thus, the positive cases can be recalled in a timely manner. Parents can also visit the website through the specified path to self-query the results for their newborn.

\section{DISCUSSION}

Our study showed that the rate of incidence of neonatal G6PD deficiency in boys was $5.20 \%$ in Guangzhou; this value is quite high. Relative higher incidence rates of G6PD deficiency have been noted in South China and southwestern region of China. The incidence rate of neonatal G6PD deficiency was found to be 5.1-7.5\% in Iran (Iranpour et al., 2008; Karimi et al., 2008). Kuwahata et al. (2010) reported that the incidence rate of neonatal G6PD deficiency in boys was $21.70 \%$ (843/1731) in the Isabel Province of Solomon Islands. G6PD deficiency is the chief reason causing neonatal hyperbilirubinemia, infant jaundice, and hemo- 
lytic anemia, which may lead to increased rates of child mortality and disability intelligence. G6PD deficiency could be a high risk factor causing delay in the treatment of neonatal jaundice (Gundur et al., 2010) and one of high risk perinatal factors in the early neonatal period (Abdel et al., 2010). Hyperbilirubinemia caused by G6PD deficiency accounted for $14.5 \%$ of 69 cases of neonatal hyperbilirubinemia (Nishimura and Ohashi, 2010). Patients with G6PD deficiency combined with other diseases such as hepatitis A were prone to autoimmune hemolytic anemia (Ozbay et al., 2008). It has been reported that specific methods are necessary for the diagnosis of G6PD deficiency, especially for patients undergoing cardiac surgery (Dogra et al., 2010). In addition, Harish Nair showed (Nair, 2009) that patients with different genotypes of G6PD have differing susceptibilities to Plasmodium falciparum (Johnson et al., 2009).

Based on 22 years of practice and experience in neonatal screening for G6PD deficiency, we believe that it is essential to carry out neonatal screening for G6PD deficiency to allow prevention and timely treatment. For the issues raised by Parashar (2010), Harish Nair showed (Nair, 2009) that G6PD deficiency is a high risk factor contributing to neonatal hyperbilirubinemia and nuclear jaundice. The rates of morbidity and mortality can be effectively reduced by neonatal screening for G6PD deficiency. Cohan showed that after the neonatal screening program, the hospitalization rate of patients with G6PD deficiency significantly decreased in southern Iran (Cohan et al., 2010). A previous study showed that it was possible to predict the extent of severity of neonatal hyperbilirubinemia by determination of G6PD enzyme activity once a patient with G6PD deficiency was identified by neonatal screening. At the global symposium on neonatal jaundice and nuclear jaundice held in Siena, Italy, in 2005 , some scholars proposed that at discharge, i) the results of screening for G6PD deficiency should be shared, ii) parents should be made aware of the implications of the disease, and iii) long-term care and follow-up treatment should be made available for the patients by the hospital (Nock et al., 2011). Requirement analysis and feasibility study of the neonatal screening program for G6PD deficiency was conducted in India in 2009 and it emphasized the need for the program to be integrated into a national screening program. In 2010, Nock reported that a pilot in-hospital newborn screening program for G6PD deficiency was carried out in the United States (Nock et al., 2011).

During the neonatal screening program for G6PD deficiency, the following problems should be resolved: i) early collection, transport, screening, and testing of specimens; ii) early and timely reporting of the results of the screening program; iii) prevention and treatment of neonatal hyperbilirubinemia and hemolytic anemia within a week of childbirth; iv) genetic counseling for healthcare of patients with G6PD deficiency. Dried bloodspot specimens for newborn screening were often collected on day 3 of birth in the neonatal screening programs of China. Early collection of specimens may increase the false-positive rate for thyroid dysfunction (hypothyroidism) and the false-negative rate for phenylketonuria (PKU). Delay in blood collection may not be conducive to the early diagnosis of G6PD deficiency. As the G6PD activity of the blood sample attenuated rapidly in vitro, the G6PD activity of the dried bloodspot reduced up to one-third at 1 week after blood collection and only $50 \%$ of the G6PD activity remained after 2 weeks. The freshness of the specimens is critical for the results. Although the desired speed of specimen delivery to the screening center is within $48 \mathrm{~h}$ of collection, the screening specimen should be submitted as soon as possible. The collection involves the use of "the green channel for the neonatal screening specimen," which was set up at Shanghai, China, in 1999. Since April 2009, in Guangzhou, China, the specimen blood 
films has been delivered using the "Half Day Delivery" or "Regional Next Morning Delivery" systems of the express mail service (EMS). Therefore, the specimens can be delivered more accurately and quickly to the screening laboratory within 4-5 days of birth. It is advisable to detect the specimen immediately after receipt at the newborn screening center to avoid natural attenuation of G6PD activity because of delayed detection by using dried bloodspot specimens and increased chances of false-positive results. Fluorescent spot test and quantitative fluorescence assay have been successfully applied in neonatal screening for G6PD deficiency by the Guangzhou Neonatal Screening Center. The principle of the 2 methods is based on the determination of the absorbance at $340 \mathrm{~nm}$ due to NADPH formation. The quantitative evaluation is done by adding a precise amount of the hemolysate to an assay mixture containing the substrate (glucose 6-phosphate) and its cofactor NADP. The rate of NADPH generation used to indicate the availability or deficiency of G6PD is spectrophotometrically measured at $340 \mathrm{~nm}$ (Minucci et al., 2009). The detection rate for the 2 methods is $100 \%$ for samples from male patients and all the female homozygous patients. The 2 methods are simple, less time consuming, and are suitable for batch testing of a large number of specimens. The former is a qualitative method and the accuracy of the results depends on the operator's observation of the fluorescent response of the reaction product. The results of the former method may be affected by subjectivity and the fluorescence data cannot be saved. The advantage of the former method is that it does not require expensive equipment. The latter is a quantitative method with high sensitivity and the results are objective and accurate. The method can improve the detection rate of heterozygotes and the experimental data can be saved for a long time. The positive results obtained for G6PD deficiency screening were further confirmed by determining the ratio of venous G6PD to 6PGD by using the modified NBT quantitative method. The patient whose G6PD activity value was below the cut-off point was diagnosed to be positive for G6PD deficiency. In addition, dried bloodspots on filter paper can also be used to analyze the point mutation of G6PD gene (Beutler and Vulliamy, 2002). Like other screening programs, G6PD screening also needs to be subjected to the inter-laboratory quality assessment to ensure quality. Since 1998, our Newborn Screening Center in the Guangzhou City has participated in the inter-laboratory quality assurance program held by Yang-Ming University, Taipei, China. The false-negative rate was zero and the false-positive rate was $1.2 \%$.

To maximize the benefits of the G6PD deficiency screening program, the screening results should be broadcast timely to create conditions conducive for the early prevention and treatment of clinical neonatal hyperbilirubinemia and hemolytic anemia. In some areas, the results are informed by telephone to the parents or physicians of the patients; this is a preferred method of notification. In the Guangzhou Neonatal Screening Center, since October 2001, the results of screening, including screening for G6PD deficiency, hypothyroidism, PKU, and other projects, were sent through the Internet network system. All positive or negative results can be transmitted real time and the results of G6PD screening can be printed through a network computer in the affiliated maternity hospitals.

Intravascular hemolysis caused by some inducing damage factors may occur easily in patients with G6PD deficiency (Luzzatto, 2006). For example, neonatal hyperbilirubinemia may be induced or exacerbated by infections, hypoxia, metabolic abnormalities (hypoglycemia, acidosis), and application of oxytocin and other perinatal factors during childbirth. G6PD deficiency is the chief reason for neonatal hyperbilirubinemia in the areas with high rate of incidence. Favism can be caused by breastfed children if the lactating mothers eat dry, fresh 
beans or bean products, including tofu and soy sauce. Acute hemolysis may be induced in patients with viral or bacterial infections such as viral respiratory infections, pneumonia, infectious hepatitis, typhoid, and septicemia, when drugs with oxidative effects such as analgesics, antipyretics, antimalarial drugs, sulfonamides, and furans are used. The preventive methods are to avoid predisposing factors in the prenatal, intrapartum, and postnatal period, to control infection, to reduce free radical damage to red blood cells, to improve fetal and neonatal liver capacity to process bilirubin, and to treat neonatal jaundice with light therapy at timely intervals. In case of neonatal hyperbilirubinemia or hemolytic anemia, patients should be treated with the routine medical treatment. In case of urgent blood transfusion, rapid detection of G6PD should be performed. Transfusion should be allowed if the test results are normal. Moreover, the transfused blood may cause hemolysis, leading to disease aggravation.

G6PD deficiency is an incomplete dominant X-linked genetic disease. This genetic disorder is transmitted from a father to his daughter and from a mother to half of her offspring. Some female heterozygous patients cannot be diagnosed because the G6PD activity value is higher than the cut-off point (Reclos et al., 2000). Lifelong healthcare precautions including regular blood test (for red blood indicators) should be undertaken by patients with G6PD deficiency to prevent hemolysis. In case of complications, the patients should not hesitate to consult a doctor.

\section{Conflicts of interest}

The authors declare no conflict of interest.

\section{REFERENCES}

Abdel FM, Abdel GE, Adel A, Mosallam D, et al. (2010). Glucose-6-phosphate dehydrogenase and red cell pyruvate kinase deficiency in neonatal jaundice cases in egypt. Pediatr. Hematol. Oncol. 27: 262-271.

Beutler E and Vulliamy TJ (2002). Hematologically important mutations: glucose-6-phosphate dehydrogenase. Blood Cells Mol. Dis. 28: 93-103.

Cohan N, Karimi M, Khalili AH, Falahzadeh MH, et al. (2010). The efficacy of a neonatal screening programme in decreasing the hospitalization rate of patients with G6PD deficiency in southern Iran. J. Med. Screen. 17: 66-67.

Dogra N, Puri GD and Rana SS (2010). Glucose-6-phosphate dehydrogenase deficiency and cardiac surgery. Perfusion 25: 417-421.

Foroughinia F and Karimi M (2007). The protective effects of Ginkgo biloba extract in decreasing hemolysis of RBC in Glucose-6-phosphate dehydrogenase-deficient patients. J. Pediatr. Hematol. Oncol. 29: 511-512.

Gundur NM, Kumar P, Sundaram V, Thapa BR, et al. (2010). Natural history and predictive risk factors of prolonged unconjugated jaundice in the newborn. Pediatr. Int. 52: 769-772.

Iranpour R, Hashemipour M, Talaei SM, Soroshnia M, et al. (2008). Newborn screening for glucose-6-phosphate dehydrogenase deficiency in Isfahan, Iran: a quantitative assay. J. Med. Screen. 15: 62-64.

Johnson MK, Clark TD, Njama-Meya D, Rosenthal PJ, et al. (2009). Impact of the method of G6PD deficiency assessment on genetic association studies of malaria susceptibility. PLoS One 4: e7246.

Karimi M, Yavarian M, Afrasiabi A, Dehbozorgian J, et al. (2008). Prevalence of beta-thalassemia trait and glucose-6phosphate dehydrogenase deficiency in Iranian Jews. Arch. Med. Res. 39: 212-214.

Kaur G, Srivastav J, Jain S, Chawla D, et al. (2010). Preliminary report on neonatal screening for congenital hypothyroidism, congenital adrenal hyperplasia and glucose-6-phosphate dehydrogenase deficiency: a Chandigarh experience. Indian J. Pediatr. 77: 969-973.

Kuwahata M, Wijesinghe R, Ho MF, Pelecanos A, et al. (2010). Population screening for glucose-6-phosphate dehydrogenase deficiencies in Isabel Province, Solomon Islands, using a modified enzyme assay on filter paper dried bloodspots. Malar. J. 9: 223.

Luzzatto L (2006). Glucose 6-phosphate dehydrogenase deficiency: from genotype to phenotype. Haematologica 91: 
1303-1306.

Minucci A, Giardina B, Zuppi C and Capoluongo E (2009). Glucose-6-phosphate dehydrogenase laboratory assay: How, when, and why? IUBMB Life 61: 27-34.

Nair H (2009). Neonatal screening program for G6PD deficiency in India: need and feasibility. Indian Pediatr. 46: 10451049.

Nishimura A and Ohashi K (2010). Risk factors of paternal depression in the early postnatal period in Japan. Nurs. Health Sci. 12: 170-176.

Nock ML, Johnson EM, Krugman RR, Di Fiore JM, et al. (2011). Implementation and analysis of a pilot in-hospital newborn screening program for glucose-6-phosphate dehydrogenase deficiency in the United States. J. Perinatol. 31: 112-117.

Ozbay HF, Ozcay F, Selda BU, Avci Z, et al. (2008). Etiology of hemolysis in two patients with hepatitis A infection: glucose-6-phosphate dehydrogenase deficiency or autoimmune hemolytic anemia. Eur. J. Pediatr. 167: 1435-1439.

Parashar Y (2010). G6PD screening is it really required? Indian Pediatr. 47: 451-452.

Reclos GJ, Hatzidakis CJ and Schulpis KH (2000). Glucose-6-phosphate dehydrogenase deficiency neonatal screening: preliminary evidence that a high percentage of partially deficient female neonates are missed during routine screening. J. Med. Screen. 7: 46-51. 